See discussions, stats, and author profiles for this publication at: https://www.researchgate.net/publication/319617080

\title{
Alternative models of DSM-5 PTSD: Examining diagnostic implications
}

Article in Psychiatry Research · September 2017

DOI: 10.1016/.psychres.2017.09.011

CITATIONS

8

6 authors, including:

Siobhan Murphy

Queen's University Belfast

40 PUBLICATIONS 346 CITATIONS

SEE PROFILE

Ask Elklit

University of Southern Denmark

279 PUBLICATIONS 5,505 CITATIONS

SEE PROFILE
READS

446

Maj Hansen

University of Southern Denmark

71 PUBLICATIONS 647 CITATIONS

SEE PROFILE

Yoke Yong Chen

University Malaysia Sarawak

10 PUBlications 48 Citations

SEE PROFILE

Some of the authors of this publication are also working on these related projects:

Intimate partner stalking in Denmark View project

Validation of the Odense Child Trauma Screening View project 


\title{
Alternative models of DSM-5 PTSD: Examining diagnostic implications
}

\author{
Siobhan Murphy ${ }^{\mathrm{a}, *}$, Maj Hansen $^{\mathrm{a}}$, Ask Elklit ${ }^{\mathrm{a}}$, Yoke Yong Chen ${ }^{\mathrm{b}}$, Siti Raudzah Ghazali ${ }^{\mathrm{b}}$, \\ Mark Shevlin ${ }^{\mathrm{c}}$ \\ a National Centre of Psychotraumatology, University of Southern Denmark, Odense, Denmark \\ ${ }^{\mathrm{b}}$ Faculty of Medicine and Health Science, Universiti Malaysia Sarawak, Sarawak, Malaysia \\ c Psychology Research Institute, Ulster University, Derry, Northern Ireland
}

\section{A R T I C L E I N F O}

\section{Keywords:}

Posttraumatic stress disorder

Confirmatory factor analysis

Diagnostic implications

PTSD Checklist for DSM-5

\begin{abstract}
A B S T R A C T
The factor structure of DSM-5 posttraumatic stress disorder (PTSD) has been extensively debated with evidence supporting the recently proposed seven-factor Hybrid model. However, despite myriad studies examining PTSD symptom structure few have assessed the diagnostic implications of these proposed models. This study aimed to generate PTSD prevalence estimates derived from the 7 alternative factor models and assess whether pre-established risk factors associated with PTSD (e.g., transportation accidents and sexual victimisation) produce consistent risk estimates. Seven alternative models were estimated within a confirmatory factor analytic framework using the PTSD Checklist for DSM-5 (PCL-5). Data were analysed from a Malaysian adolescent community sample $(n=481$ ) of which $61.7 \%$ were female, with a mean age of 17.03 years. The results indicated that all models provided satisfactory model fit with statistical superiority for the Externalising Behaviours and seven-factor Hybrid models. The PTSD prevalence estimates varied substantially ranging from $21.8 \%$ for the DSM-5 model to $10.0 \%$ for the Hybrid model. Estimates of risk associated with PTSD were inconsistent across the alternative models, with substantial variation emerging for sexual victimisation. These findings have important implications for research and practice and highlight that more research attention is needed to examine the diagnostic implications emerging from the alternative models of PTSD.
\end{abstract}

\section{Introduction}

The underlying latent structure of posttraumatic stress disorder (PTSD) has been extensively studied and debated (Armour et al., 2016). Most research into PTSD symptoms in the Diagnostic and Statistical Manual for Mental Disorders (American Psychiatric Association, 2013) has supported and demonstrated superior fit of two alternative four factor models over the three factor DSM-IV PTSD model; the Emotional Numbing model (King et al., 1998), and the Dysphoria model (Elhai and Palmieri, 2011; Simms et al., 2002; Yufik and Simms, 2010). More recently, Elhai et al. (2011) proposed a five factor Dysphoric Arousal model which spilt the hyperarousal symptom cluster into dysphoric and anxious arousal symptoms. This separation was based on evidence documenting the difference between general distress/dysphoria (D1D3) and fear based symptoms (D4-D5) (Watson, 2005, 2009).

The current DSM-5 conceptualisation of PTSD is more closely aligned to the Emotional Numbing model and includes four symptom clusters; re-experiencing, avoidance, negative alterations in cognitions and mood (NACM), and alterations in arousal and reactivity. Notable differences in the DSM-5 criteria are evidenced by the narrowing definition of what constitutes a traumatic event in criterion A; the removal of criterion A2 (i.e., the peri-traumatic fear, helplessness, or horror); the separation of the DSM-IV Criterion C of active avoidance and emotional numbing into two separate clusters; and the addition of three symptoms; blame of self or others, persistent negative emotions, and reckless or self-destructive behaviour (Weathers, 2017).

Following the release of the DSM-5 new evidence emerged suggesting that the factor structure of PTSD is better conceptualised as six factors; namely, the Anhedonia model (Liu et al., 2014) and Externalising Behaviours model (Tsai et al., 2015). The most recent model is a seven-factor Hybrid model (Armour et al., 2015) which has generated superior empirical support across multiple studies (Armour et al., 2016). This hybrid model integrates features of both 6 factor models including the re-experiencing, avoidance, externalising behaviours, anxious arousal and dysphoric arousal factors (from the externalising behaviours model), and the anhedonia and NACM factors (from the anhedonia model). Collectively, evidence suggests an overall tendency for the Dysphoric Arousal model to provide superior support for DSM-IV symptoms and the Hybrid model to be superior for DSM-5 symptoms (Armour et al., 2016).

\footnotetext{
* Corresponding author.

E-mail address: smurphy@health.sdu.dk (S. Murphy).
} 
Importantly, despite the theoretical and empirical support for each PTSD model there are several questions that emerge in terms of the implications on research and clinical practice. Firstly, there is a lack of knowledge about the impact of these models on diagnostic criteria and PTSD prevalence, as none of these studies have provided a diagnostic algorithm to base the diagnosis on. Considering these concerns, a recent study developed diagnostic algorithms for the seven existing DSM-5 models to examine if PTSD prevalence varied according to the different models in a clinical sample with a history of traumatic exposure (Shevlin et al., 2017). These algorithms were developed under the guidelines outlined in the DSM-5 whereby clusters containing 2-5 symptoms required the presence of at least 1 symptom (Criteria $B$ and C). The same logic followed for Criteria D and E whereby any cluster containing 6 or more symptoms, a minimum of 2 symptoms was required. Notably, the Dysphoria model deviated from these guidelines whereby a minimum of 3 symptoms were required for the dysphoria cluster. Shevlin and colleagues (2017) justified this requirement due to the higher number of symptoms (i.e. 11 symptoms) that fell into this cluster and also to coincide with the 6 symptoms required for the DSM5 PTSD diagnosis. The results of this study found significant variation in prevalence estimates with the highest estimate generated by the DSM- 5 model $(83.9 \%)$ and the lowest from the Hybrid model (64.5\%). Furthermore, Shevlin et al. (2017) also examined whether the relationship between known risk factors associated with PTSD remained consistent irrespective of which diagnostic algorithm was applied. Findings indicated that the odds ratios for an estimated PTSD diagnosis following childhood maltreatment varied between 1.89 and 3.50 for the Hybrid model and the DSM-5 model respectively. Shevlin et al. (2017) concluded that it is unclear which estimate is correct, but the magnitude of variation in child maltreatment as a risk factor for PTSD raises important implications for whether PTSD is being consistently diagnosed across all models.

The current study therefore aims to replicate and expand the findings of Shevlin and colleagues using a community sample of Malaysian adolescents. The first aim was to generate prevalence estimates from the existing seven PTSD models. The fit of these seven models were estimated which included; the 4-factor DSM-5 model, the 4-factor Dysphoria model, the 5-factor Dysphoric Arousal model, the 6-factor Anhedonia model, the 6-factor Externalising Behaviours model, the 6factor Alternative Dysphoria model and the 7-factor Hybrid model (see Table 1 for model specifications). Based on previous research (Armour et al., 2016), we hypothesised that the 7-factor Hybrid model would provide the best fit to the data. The second aim was to extend the findings of the Shevlin and colleagues study by examining whether a broader range of traumatic exposures were differentially associated with PTSD depending on the model used to derive the diagnosis. Based on literature documenting risk factors for PTSD we examined whether exposure to a natural disaster (e.g., Cao et al., 2003; Galea et al., 2007; Neria et al., 2008), transportation accidents (e.g., Murray et al., 2002), childhood neglect and sexual victimisation (e.g., Fergusson et al., 2013; Jonas et al., 2011) conferred relatively similar estimates of risk irrespective of which model of PTSD is used.

\section{Method}

\subsection{Participants and procedure}

The participants included in the present study are part of a wider project designed to examine the association between trauma exposure and physical health problems following a recent natural disaster (flood) in Malaysia $(\mathrm{N}=731)$. The data used in the present study consists of 589 adolescents aged 15-19 years with a mean age of 16.98 (SD = 1.20). The majority of the sample $373(63.3 \%)$ were females and 216 $(36.7 \%)$ were males. Ethnicity was self-reported as predominantly Malays $455(77.2 \%)$ and the remaining participants were Chinese, Indian, Bidayuh, and Ibans. The majority of the sample were still living with both parents $(81.3 \%)$ with $44(7.5 \%)$ living with one parent and the remaining participants lived with relatives or attended boarding school. Participants were recruited based on multistage sampling. Participants were contacted through the head of the villages and the school administrations. All participants provided written consent for participation and permission for underage participants was obtained from parents or legal guardians. Ethical approval was endorsed by the Ethic Committees, Faculty of Medicine and Health Sciences, Universiti Malaysia Sarawak. Permission to conduct the study was obtained from the Malaysian Ministry of Education and the Malaysian Ministry of Health.

\subsection{Measures}

\subsubsection{Traumatic exposure}

Participants were presented with a list of traumatic and negative life

Table 1

Item mappings of the alternative models of DSM-5 PTSD.

\begin{tabular}{|c|c|c|c|c|c|c|c|}
\hline Symptom & DSM-5 & Dysphoria & Dysphoric Arousal & $\begin{array}{l}\text { Externalising } \\
\text { Behaviours }\end{array}$ & Anhedonia & $\begin{array}{l}\text { Alternative } \\
\text { Dysphoria }\end{array}$ & Hybrid \\
\hline B1. Intrusive thoughts & $\mathrm{R}$ & $\mathrm{R}$ & $\mathrm{R}$ & $\mathrm{R}$ & $\mathrm{R}$ & $\mathrm{R}$ & $\mathrm{R}$ \\
\hline B2. Nightmares & $\mathrm{R}$ & $\mathrm{R}$ & $\mathrm{R}$ & $\mathrm{R}$ & $\mathrm{R}$ & $\mathrm{R}$ & $\mathrm{R}$ \\
\hline B3. Flashbacks & $\mathrm{R}$ & $\mathrm{R}$ & $\mathrm{R}$ & $\mathrm{R}$ & $\mathrm{R}$ & $\mathrm{R}$ & $\mathrm{R}$ \\
\hline B4. Emotional cue reactivity & $\mathrm{R}$ & $\mathrm{R}$ & $\mathrm{R}$ & $\mathrm{R}$ & $\mathrm{R}$ & $\mathrm{R}$ & $\mathrm{R}$ \\
\hline B5. Physiological cue reactivity & $\mathrm{R}$ & $\mathrm{R}$ & $\mathrm{R}$ & $\mathrm{R}$ & $\mathrm{R}$ & $\mathrm{R}$ & $\mathrm{R}$ \\
\hline C1. Avoidance of thoughts & A & A & $\mathrm{A}$ & A & A & $\mathrm{A}$ & A \\
\hline C2. Avoidance of reminders & A & A & A & A & A & $\mathrm{A}$ & A \\
\hline D1. Trauma-related amnesia & NACM & $\mathrm{D}$ & NACM & NACM & NACM & $\mathrm{D}$ & NA \\
\hline D2. Negative beliefs & NACM & $\mathrm{D}$ & NACM & NACM & NACM & $\mathrm{D}$ & NA \\
\hline D3. Blame of self or others & NACM & $\mathrm{D}$ & NACM & NACM & NACM & $\mathrm{D}$ & NA \\
\hline D4. Negative trauma related emotions & NACM & $\mathrm{D}$ & NACM & NACM & NACM & $\mathrm{D}$ & NA \\
\hline D5. Loss of interest & NACM & $\mathrm{D}$ & NACM & NACM & AN & AN & AN \\
\hline D6. Detachment & NACM & $\mathrm{D}$ & NACM & NACM & AN & AN & AN \\
\hline D7. Restricted affect & NACM & $\mathrm{D}$ & NACM & NACM & AN & AN & AN \\
\hline E1. Irritability/anger & $\mathrm{AR}$ & $\mathrm{D}$ & $\mathrm{DA}$ & $\mathrm{EB}$ & DA & $\mathrm{EB}$ & $\mathrm{EB}$ \\
\hline E2. Self-destructive/reckless behaviour & AR & $\mathrm{AR}$ & DA & EB & DA & EB & EB \\
\hline E3. Hypervigilance & AR & $\mathrm{AR}$ & AA & AA & AA & AA & AA \\
\hline E4. Exaggerated startle response & $\mathrm{AR}$ & $\mathrm{AR}$ & $\mathrm{AA}$ & AA & $\mathrm{AA}$ & $\mathrm{AA}$ & AA \\
\hline E5. Difficulty concentrating & $\mathrm{AR}$ & $\mathrm{D}$ & DA & DA & DA & $\mathrm{D}$ & DA \\
\hline E6. Sleep disturbance & $\mathrm{AR}$ & $\mathrm{D}$ & DA & DA & DA & $\mathrm{D}$ & DA \\
\hline
\end{tabular}

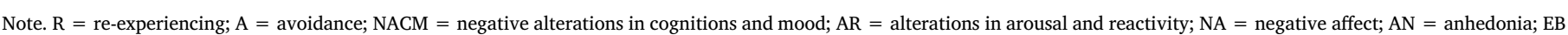
= externalising behaviours; $\mathrm{DA}=$ dysphoric arousal. 
Table 2

Number of symptoms required from each cluster for each PTSD symptom profile for diagnostic purposes.

\begin{tabular}{|c|c|c|c|c|c|c|c|}
\hline PTSD symptom cluster & DSM-5 & Dysphoria & Dysphoric Arousal & Anhedonia & Externalising Behaviours & $\begin{array}{l}\text { Alternative } \\
\text { Dysphoria }\end{array}$ & Hybrid \\
\hline Intrusions & $1 / 5$ & $1 / 5$ & $1 / 5$ & $1 / 5$ & $1 / 5$ & $1 / 5$ & $1 / 5$ \\
\hline Avoidance & $1 / 2$ & $1 / 2$ & $1 / 2$ & $1 / 2$ & $1 / 2$ & $1 / 2$ & $1 / 2$ \\
\hline NACM & $2 / 7$ & - & $2 / 7$ & $1 / 4$ & $2 / 7$ & - & $1 / 4$ \\
\hline Hyperarousal & $2 / 6$ & $1 / 2$ & - & - & - & - & - \\
\hline Dysphoria & - & $3 / 11$ & - & - & - & $2 / 6$ & - \\
\hline Dysphoric Arousal & - & - & $1 / 4$ & $1 / 4$ & $1 / 2$ & - & $1 / 2$ \\
\hline Anxious Arousal & - & - & $1 / 2$ & $1 / 2$ & $1 / 2$ & $1 / 2$ & $1 / 2$ \\
\hline Anhedonia & - & - & - & $1 / 3$ & - & $1 / 3$ & $1 / 3$ \\
\hline Externalising Behaviours & - & - & - & - & $1 / 2$ & $1 / 2$ & $1 / 2$ \\
\hline Total symptoms required & $6 / 20$ & $6 / 20$ & $6 / 20$ & $6 / 20$ & $7 / 20$ & $7 / 20$ & $7 / 20$ \\
\hline
\end{tabular}

events. Thirteen items were derived from the Life Events Checklist for DSM-5 (Weathers et al., 2013a). These questions included direct and indirect exposure to a natural disaster, transportation accident and sexual assault. An additional five items were added to capture events e.g., near drowning experiences, robbery, and childhood neglect. Participants were also asked to endorse their most traumatic event. The data was then screened to ensure that only participants who reported traumatic exposure were included. Of the full adolescent sample, 108 participants did not report traumatic exposure and were therefore removed from the analyses, leaving a final sample of 481 . The majority of this sample were female $61.7 \%$ with a mean age of 17.03 years. Four items were selected from this checklist to examine the association of pre-established risk factors for PTSD which included; exposure to a natural disaster, experiencing a transportation accident, childhood neglect and lifetime sexual victimisation (sexual assault or rape).

\subsubsection{PTSD Checklist for DSM-5 (PCL-5; Weathers et al., 2013b)}

PTSD was assessed using Malay language version of the PCL-5. The PCL-5 consists of 20 items that correspond to the DSM-5 PTSD symptoms. Participants are asked to indicate "how much have you been bothered by the problem in the past month" and responses are rated on a five-point Likert scale $(0=$ Not at all, $1=$ A little bit, $2=$ Moderately, 3 = Quite a bit, 4 = Extremely). To establish diagnostic rates the DSM-5 algorithm was applied which requires at least; one intrusion item (B1-B5), one avoidance item (C1-C2), two items from the negative alternations in cognition and mood (NACM; D1-D7) and two hyperarousal items (E1-E6). A rating of 2 (i.e. moderately) or higher for an item is treated as the presence of a symptom. The psychometric properties of the PCL- 5 have been assessed across a variety of traumaexposed samples and the scale has demonstrated satisfactory reliability and validity (e.g., Blevins et al., 2015; Bovin et al., 2015). In the current sample, the internal reliability for the full scale was satisfactory ( $\alpha=$ 0.91 ), and each subscale: intrusions ( $\alpha=0.80$ ), avoidance ( $\alpha=0.73$ ), NACM $(\alpha=0.85)$, and hyperarousal $(\alpha=0.77)$.

\subsection{Statistical analysis}

The first stage of the analysis was to assess the latent structure of the PCL-5 using confirmatory factor analysis (CFA) based on responses to the 20 items. Seven models were specified and estimated by Mplus 7.1 (Muthen and Muthen, 2013) using the robust weighted least squares estimator (WLSMV) based on the polychoric correlation matrix of latent continuous response variables and robust maximum likelihood estimation (MLR: Yuan and Bentler, 2000). The models are presented in Table 1.

In order to assess the goodness of fit for each model using both estimators a range of fit statistics were examined including; the comparative fit index (CFI; Bentler, 1990), and the Tucker-Lewis Index (TLI; Tucker and Lewis, 1973), a non-significant $\chi^{2}$ and Root Mean Square Error of Approximation (RMSEA; Steiger, 1990). Specifically, a CFI/TLI above 0.95 indicate a good fit between the model and the data. A moderate fit is indicated by a CFI above 0.90 . Additionally, the RMSEA of where a value less than 0.05 indicate close fit and values up to 0.08 indicate reasonable errors of approximation. The Weighted Root Mean Square Residual (WRMR) was inspected when using the WLMSV estimator whereby values less than 1 are indicative of acceptable model fit. For MLR estimation the Standardised Root-Mean Square Residual (SRMR: Joreskog and Sorbom, 1996) was used with values of less than 0.06 indicating excellent fit and values less than 0.08 indicative of acceptable model fit. The Bayesian Information Criterion (BIC: Schwarz, 1978) was used to evaluate and compare models, with the smallest value indicating the best fitting model. In examining BIC differences, it has been suggested that a difference of $6-10$ indicates strong evidence of model superiority and a difference $>10$ indicates very strong evidence of model superiority (Raftery, 1996).

The second phase of the analysis involved generating probable selfreported prevalence rates of PTSD for each model based on a score of 2 (moderately) or greater being indicative of a symptom present. The symptom-based diagnostic algorithm for each model was developed by Shevlin et al. (2017) and are presented in Table 2.

The third phase of the analysis was to explore a range of traumatic experiences that have been associated with the development of PTSD (i.e., experiencing a natural disaster, a road traffic accident, childhood neglect and sexual victimisation) to determine whether differential associations emerged between alternative forms of traumatic exposure and PTSD rates as derived from the alternative models of DSM-5 PTSD symptoms.

\section{Results}

A total of 364 (75.7\%) participants reported either direct or indirect exposure to a natural disaster, $305(63.4 \%)$ reported experiencing a transportation accident, 33 (6.9\%) reported experiencing childhood neglect and $10(2.1 \%)$ endorsed lifetime sexual victimisation. There were no significant gender differences between exposure to a natural disaster $\chi^{2}(1)=0.07, p=0.79$; sexual victimisation $\chi^{2}(1)=0.13, p$ $=0.91$ or childhood neglect $\chi^{2}(1)=0.95 p=0.33$. Males were significantly more likely to endorse a transportation accident $\chi^{2}(1)=$ $12.74, p<0.001$.

The fit statistics for the seven competing CFA models are presented in Table 3. Although the chi-square statistics were statistically significant this should not lead to the rejection of the models as the large sample size increased the power of the test (Tanaka, 1987). Using WLMSV estimation all models met the criteria for an excellent model fit based on the CFI and TLI, and RMSEA criteria. For models based on MLR estimation all demonstrated acceptable model fit with the Hybrid model providing the lowest BIC value. We further examined the BIC differences from the Anhedonia and Externalising Behaviours and found that the Hybrid was marginally superior for the Anhedonia based on BIC difference of 10.97 but not for the Externalising Behaviours model with a BIC difference of 5.03. The estimated diagnostic rates corresponding to the seven symptom algorithms are also presented in 
Table 3

Model fit statistics for alternative models of DSM-5 PTSD and prevalence rates based on each symptom algorithm.

\begin{tabular}{|c|c|c|c|c|c|c|c|c|c|}
\hline Model & Estimator & Chi Square & Df & CFI & TLI & RMSEA & $\begin{array}{l}\text { WRMR } \\
\text { SRMR }\end{array}$ & BIC & DX\% \\
\hline \multirow[t]{2}{*}{ DSM -5} & WLSMV & 452.43 & 164 & 0.96 & 0.95 & $\begin{array}{l}0.06 \\
0.54-0.67\end{array}$ & 1.10 & & 21.8 \\
\hline & MLR & 337.39 & 164 & 0.94 & 0.93 & $\begin{array}{l}0.047 \\
0.040-0.054\end{array}$ & 0.49 & $25,344.01$ & \\
\hline \multirow[t]{2}{*}{ Dysphoria } & WLSMV & 508.29 & 164 & 0.95 & 0.94 & $\begin{array}{l}0.07 \\
0.06-0.07\end{array}$ & 1.18 & & 20.0 \\
\hline & MLR & 391.86 & 164 & 0.92 & 0.90 & $\begin{array}{l}0.054 \\
0.05-0.06\end{array}$ & 0.048 & $25,415.78$ & \\
\hline \multirow[t]{2}{*}{ Dysphoric Arousal } & WLSMV & 408.16 & 160 & 0.97 & 0.96 & $\begin{array}{l}0.057 \\
0.05-0.06\end{array}$ & 1.02 & & 18.7 \\
\hline & MLR & 311.02 & 160 & 0.94 & 0.93 & $\begin{array}{l}0.044 \\
0.04-0.05\end{array}$ & 0.04 & $25,331.83$ & \\
\hline \multirow[t]{2}{*}{ Anhedonia } & WLSMV & 375.01 & 155 & 0.97 & 0.96 & $\begin{array}{l}0.052 \\
0.05-0.06\end{array}$ & 0.96 & & 15.2 \\
\hline & MLR & 283.81 & 155 & 0.95 & 0.94 & $\begin{array}{l}0.042 \\
0.03-0.05\end{array}$ & 0.04 & $25,325.11$ & \\
\hline \multirow[t]{2}{*}{$\begin{array}{l}\text { Externalising } \\
\text { Behaviours }\end{array}$} & WLSMV & 370.06 & 155 & 0.97 & 0.96 & $\begin{array}{l}0.05 \\
0.05-0.06\end{array}$ & 0.963 & & 11.2 \\
\hline & MLR & 278.42 & 155 & 0.95 & 0.94 & $\begin{array}{l}0.04 \\
0.33-0.048\end{array}$ & 0.04 & $25,319.17$ & \\
\hline \multirow[t]{2}{*}{ Alternative Dysphoria } & WLSMV & 422.04 & 155 & 0.96 & 0.95 & $\begin{array}{l}0.06 \\
0.05-0.07\end{array}$ & 1.04 & & 12.9 \\
\hline & MLR & 325.25 & 155 & 0.93 & 0.92 & $\begin{array}{l}0.05 \\
0.04-0.06\end{array}$ & 0.05 & $25,380.25$ & \\
\hline \multirow[t]{2}{*}{ Hybrid } & WLSMV & 326.26 & 149 & 0.98 & 0.97 & $\begin{array}{l}0.05 \\
0.04-0.06\end{array}$ & 0.88 & & 10.0 \\
\hline & MLR & 247.966 & 149 & 0.96 & 0.95 & $\begin{array}{l}0.037 \\
0.03-0.05\end{array}$ & 0.04 & $25,314.14$ & \\
\hline
\end{tabular}

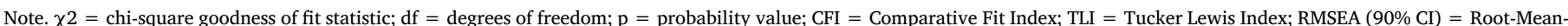

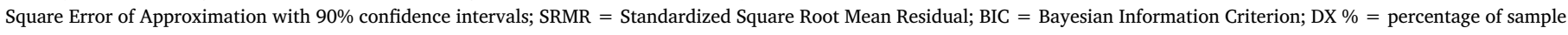
meeting "diagnosis" according to each symptom profile.

Table 3. The seven symptom profiles demonstrated considerable variation, with the DSM-5 model producing the highest diagnostic rates (21.8\%) and the Hybrid model producing the lowest (10.0\%). The difference in the two models was statistically significant $(z=5.45$, $p<0.05$ ). In comparing the prevalence estimates derived from the DSM-5 and the Anhedonia (15.2\%) and Externalising Behaviours (11.2\%) models there was a significant difference $(z=2.64, p$ $=<0.05)$ and $(z=4.43, p=<0.05)$ respectively. There was a significant difference in prevalence estimates for the Anhedonia and the Hybrid models $(z=2.43, p<0.05)$ however, not for the Externalising Behaviour and the Hybrid models $(z=0.60, p>0.05)$.

Table 4 presents the results of the bivariate associations between four types of traumatic exposure and the seven PTSD models. Exposure to a natural disaster was not significantly associated with a PTSD diagnosis in any of the models, whilst, experiencing a road traffic accident was significantly associated with PTSD in all models except the Alternative Dysphoria model. Sexual victimisation was significantly associated with PTSD in all models, however the magnitude in risk varied depending on each model with odds ratios ranging from 3.71 for the DSM-5 model to 6.47 for the Hybrid model. Notably, childhood maltreatment was only significantly associated with PTSD in the Anhedonia model and marginally significant for the Dysphoria model.

Table 4

Bivariate associations between trauma variables and risk of diagnosis for each model.

\begin{tabular}{|c|c|c|c|c|c|c|c|c|c|c|c|c|}
\hline \multirow[b]{2}{*}{ Model } & \multicolumn{3}{|c|}{ Natural Disaster } & \multicolumn{3}{|c|}{ Road Traffic Accident } & \multicolumn{3}{|c|}{ Childhood Neglect } & \multicolumn{3}{|c|}{ Sexual Victimisation } \\
\hline & $\mathbf{N}$ & $\chi^{2}(\mathrm{df})$ & OR & $\mathbf{N}$ & $\chi^{2}(\mathrm{df})$ & OR & $\mathbf{N}$ & $\chi^{2}(\mathrm{df})$ & OR & $\mathbf{N}$ & $\chi^{2}(\mathrm{df})$ & OR \\
\hline & $\%$ & $\mathrm{p}$ & $95 \% \mathrm{CI}$ & $\%$ & $\mathrm{p}$ & $95 \% \mathrm{CI}$ & $\%$ & $\mathrm{p}$ & $95 \% \mathrm{CI}$ & $\%$ & $\mathrm{p}$ & $95 \% \mathrm{CI}$ \\
\hline \multirow[t]{2}{*}{ DSM -5} & 77 & $0.40(1)$ & 0.85 & 79 & $8.10(1)$ & 2.02 & 11 & $2.75(1)$ & 1.88 & 5 & $4.75(1)$ & 3.71 \\
\hline & 21.2 & 0.53 & $(0.52-1.40)$ & 25.9 & 0.004 & $(1.24-3.29)$ & 33.3 & 0.09 & $(0.88-4.02)$ & 50.0 & 0.03 & (1.05-13.07) \\
\hline \multirow[t]{2}{*}{ Dys } & 69 & $0.94(1)$ & 0.80 & 72 & $6.94(1)$ & 1.70 & 11 & $3.97(1)$ & 2.14 & 5 & $5.77(1)$ & 4.18 \\
\hline & 19.0 & 0.33 & $(0.48-1.29)$ & 23.6 & 0.001 & $(1.07-2.70)$ & 33.3 & 0.05 & $(0.99-4.57)$ & 50.0 & 0.02 & (1.18-14.73) \\
\hline \multirow[t]{2}{*}{ DA } & 65 & $0.72(1)$ & 0.80 & 69 & $8.39(1)$ & 2.16 & 10 & $3.13(1)$ & 2.00 & 5 & $6.57(1)$ & 4.54 \\
\hline & 17.9 & 0.40 & $(0.48-1.34)$ & 22.6 & 0.004 & $(1.27-3.66)$ & 30.3 & 0.08 & $(0.92-4.37)$ & 50.0 & 0.01 & $(1.29-16.04)$ \\
\hline \multirow[t]{2}{*}{ Anhed } & 50 & $2.41(1)$ & 0.68 & 56 & $6.56(1)$ & 2.00 & 9 & $4.03(1)$ & 2.25 & 4 & $4.89(1)$ & 3.88 \\
\hline & 13.7 & 0.12 & $(0.41-1.12)$ & 18.4 & 0.01 & (1.15-3.39) & 27.3 & 0.04 & $(1.00-5.06)$ & 40.0 & 0.03 & $(1.07-14.12)$ \\
\hline \multirow[t]{2}{*}{ EB } & 37 & $1.69(1)$ & 0.66 & 41 & $4.11(1)$ & 1.95 & 5 & $0.55(1)$ & 1.45 & 4 & $8.48(1)$ & 5.61 \\
\hline & 10.2 & 0.19 & $(0.36-1.23)$ & 13.4 & 0.04 & $(1.01-3.74)$ & 15.2 & 0.46 & $(0.54-3.94)$ & 40.0 & 0.004 & (1.53-20.57) \\
\hline \multirow[t]{2}{*}{ ADYS } & 42 & 2.43 & 0.63 & 46 & $3.57(1)$ & 1.78 & 6 & $0.88(1)$ & 1.56 & 4 & $6.69(1)$ & 4.75 \\
\hline & 11.5 & 0.12 & $(0.36-1.13)$ & 15.1 & 0.06 & $(0.97-3.24)$ & 18.2 & 0.35 & $(0.62-3.93)$ & 40.0 & 0.001 & $(1.30-17.33)$ \\
\hline \multirow[t]{2}{*}{ Hybrid } & 32 & $2.35(1)$ & 0.62 & 37 & $4.30(1)$ & 2.04 & 5 & $1.06(1)$ & 1.68 & 4 & $10.25(1)$ & 6.47 \\
\hline & 8.8 & 0.13 & $(0.35-1.11)$ & 12.1 & 0.04 & $(1.06-3.90)$ & 15.2 & 0.30 & $(0.62-4.58)$ & 40.0 & 0.001 & $(1.76-23.80)$ \\
\hline
\end{tabular}

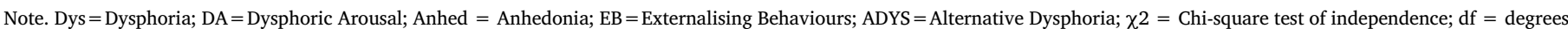
of freedom; $\mathrm{p}=$ statistical significance; OR $(95 \% \mathrm{CI})=$ odds ratio with $95 \%$ confidence intervals. 


\section{Discussion}

The overall aim of this study was to examine diagnostic rates of PTSD based on the existing factor analytical models in a sample of Malaysian adolescents exposed to a recent natural disaster and a range of other traumatic events. Firstly, seven alternative DSM-5 PTSD models were estimated and findings revealed that all models provided good fit to the data, with the Externalising Behaviours and Hybrid model providing statistical superiority. The current findings support those reported by Shevlin and colleagues (2017) and demonstrate considerable variation in diagnostic rates derived from the different models. Further, both studies found that the highest diagnostic rates were evident from the DSM-5 model and lowest rates were seen in the Hybrid model. Findings indicated that $21.8 \%$ of the sample met the diagnostic threshold for PTSD using the DSM-5 model compared to $10.0 \%$ in the Hybrid model, representing a $54.1 \%$ reduction in prevalence between the two models. The Externalising Behaviours model, Alternative Dysphoria model, and the Anhedonia model further signified a substantial reduction in PTSD prevalence equating to $48.6 \%$, $40.8 \%$ and $30.3 \%$ respectively relative to the DSM- 5 model. The reduction in prevalence for the Dysphoric Arousal and the Dysphoria models were attenuated but still noteworthy with $14.2 \%$ and $8.3 \%$ respectively.

The issue of different conceptualisations of PTSD has been debated since its inclusion in the DSM-III. Prior to the release of the DSM-5 and the suggested modifications to the diagnostic criteria, several researchers investigated the impact these different conceptualisations would have on prevalence estimates. For example, Elhai and colleagues (2012) found that PTSD prevalence was higher using the DSM-5 versus the DSM-IV criteria, however, not significantly higher in a sample of college students. Conversely, Kilpatrick et al. (2013) did find that prevalence estimates for lifetime PTSD were significantly different using DSM-5 compared to DSM-IV criteria $(9.4 \%$ and $10.6 \%$ respectively). Notably, the differences in these prevalence estimates were small. When these studies are compared to the current findings it is evident that the increasingly complex models of PTSD (e.g., the Hybrid model) adds substantial variation to the overall prevalence. For example, our findings show that over $50 \%$ of those who met the DSM- 5 criteria do not meet the criteria derived from the Hybrid model. This draws attention to the clinical implications which these existing models may have on traumatised individuals accessing and receiving treatment. It appears that the Hybrid model places a stronger focus on diagnostic specificity which aims to reduce the rate of false positive diagnoses but increases the risk of false negative diagnoses by placing stronger restrictions on the presence of certain symptoms to meet the diagnostic threshold. Conversely, the DSM-5 model focuses on diagnostic sensitivity which aims at reducing false negative diagnoses but increases the risk of false positive diagnoses. Prior to the release of the DSM- 5 the proposed changes to the diagnostic criteria of PTSD and indeed a number of other disorders, faced criticism in terms of increasing the diagnostic sensitivity and reducing the specificity (Batstra and Frances, 2012; Frances and Nardo, 2013). The most obvious risk associated with focusing more on diagnostic sensitivity is that increases the likelihood of inflated diagnoses of PTSD which subsequently may result in the prescription of unnecessary and potentially harmful treatment (Frances and Nardo, 2013). However, focusing on diagnostic specificity can also have harmful effects by increasing the likelihood of many individuals not receiving a PTSD diagnosis and restricting their recovery process. This raises a challenge for future research as based on the current findings and those of Shevlin and colleagues, it is evident that even small changes to diagnostic criteria can have substantial implications on possible rates of misdiagnosis as PTSD prevalence appears markedly different according to which model is used. It is important therefore that future research aims at reconciling the diagnostic implications based on these models and work towards achieving consensus on what accurately represents the disorder. Consequently, it is pertinent that researchers move beyond replicating these alternative factor structures in a variety of different populations and rather examine the implications that these models actually propose in clinical and research settings.

Furthermore, when comparing all the DSM-5 models to the upcoming ICD-11 the complexity of these issues are exemplified. The upcoming ICD-11 proposes to simplify the structure of PTSD into three factors; re-experiencing, avoidance and hyperarousal, all of which have two symptoms. This narrower definition is intended to direct clinicians to the core elements of the disorder and use functional impairment rather than a specific traumatic experience to determine diagnostic threshold (Maercker et al., 2013) which greatly contrasts to all models representing DSM-5 PTSD. Previous studies have demonstrated a tendency for the DSM-5 symptom profile to result in significantly higher estimated PTSD rates following different forms of traumatic exposure (cf. Hansen et al., 2015; Hyland et al., 2016; O'Donnell et al., 2014). However, other studies have not identified significant differences (Stein et al., 2014). Future studies examining the prevalence estimates in the more recent DSM- 5 factor models and ICD-11 proposed criteria would be of interest.

When examining the diagnostic accuracy of specified models, other factors need to be considered; for example, whether previously established risk factors confer relatively similar risk estimates. Using data from the World Mental Health Surveys, Stein et al. (2014) examined whether the four conceptualisations of PTSD (i.e., DSM-IV, DSM-5, ICD10 and the proposed ICD-11) were differentially associated with a range of sociodemographic and trauma-related factors. They found little evidence of significant differences across the different systems indicating that these alterations did not affect the underlying risk profile. As a secondary aim of this study we also examined this issue and found mixed evidence. Findings indicated that exposure to a road traffic accident was not significantly associated with a PTSD diagnosis in any of the models although the magnitude of the risk estimate was relatively similar. Moreover, it is noteworthy that childhood neglect was only a significant predictor of a PTSD diagnosis in the Anhedonia model and marginally significant for the Dysphoria model. Finally, in terms of sexual victimisation, whilst being a significant predictor for a PTSD diagnosis in all models, the magnitude of this risk differed substantially depending on which model was used with odds ratios ranging from 3.71 to 6.47 for the DSM-5 and Hybrid models respectively. These findings support those reported in the Shevlin and colleagues study that also found substantial variation in the magnitude of risk associated with childhood maltreatment and each of the alternative PTSD models. Future research is therefore clearly warranted to examine the association between established PTSD risk factors and the alternative factor models.

The findings of this study should be considered in light of some methodological limitations. Firstly, there are many different ways the diagnostic algorithms could have been developed and applied, therefore alternative specifications may generate different diagnostic rates. Secondly, the sample is based on a Malaysian adolescent population following a recent natural disaster which may explain the higher rates of PTSD in this study compared to other community samples. Thirdly, the analyses were based on self-reported PTSD symptoms and not by a clinician administered scale which may have inflated the current prevalence estimates. Fourthly, we were unable to examine if PTSD rates using each of the alternative models displayed similar clinical characteristics and comorbidity. Finally, the cell counts for sexual victimisation were small, and the confidence intervals were large, meaning that the resulting estimates may not be reliable. Findings relating to sexual victimisation and PTSD should be interpreted with this in mind.

To conclude, this study has supported previous theoretical and empirical findings that identify seven dimensions of PTSD that have been replicated across different samples and cultures. Overall, this study found considerable variation between prevalence rates and the alternative factor analytic models of PTSD, ranging from $22.8 \%$ to 
$10.4 \%$. Additionally, we found that the relationship between different types of traumatic experiences were differentially associated with a PTSD diagnosis based on the diagnostic algorithm applied in the current study. Future research should focus on different correlates and risk factors of the outlined factors that may advance our understanding regarding the conceptualisation of PTSD.

\section{Funding}

This research was funded by the Research Acculturation Collaborative Effort (RACE) - RACE/e(1)/888/2012 (06) by Ministry of Higher Education of Malaysia.

\section{References}

American Psychiatric Association, 2013. Diagnostic and Statistical Manual of Mental Disorders, 5th ed. American Psychiatric Association, Washington, DC.

Armour, C., Tsai, J., Durham, T.A., Charak, R., Biehn, T.L., Elhai, J.D., et al., 2015. Dimensional structure of DSM-5 posttraumatic stress symptoms: support for a hybrid Anhedonia and Externalizing Behaviors model. J. Psychiat. Res. 61, 106-113.

Armour, C., Múllerová, J., Elhai, J.D., 2016. A systematic literature review of PTSD's latent structure in the diagnostic and statistical manual of mental disorders: DSM-IV to DSM-5. Clin. Psychol. Rev. 44, 60-74.

Batstra, L., Frances, A., 2012. Diagnostic inflation: causes and a suggested cure. J. Ner. Men. Dis. 200, 474-479.

Bentler, P.M., 1990. Comparative fit indexes in structural models. Psychol. Bull. 107, 238-246.

Blevins, C.A., Weathers, F.W., Davis, M.T., Witte, T.K., Domino, J.L., 2015. The Posttraumatic Stress Disorder Checklist for DSM-5 (PCL-5): development and initial psychometric evaluation. J. Trauma. Stress. 28, 489-498.

Bovin, M.J., Marx, B.P., Weathers, F.W., Gallagher, M.W., Rodriguez, P., Schnurr, P.P., et al., 2015. Psychometric properties of the PTSD checklist for diagnostic and statistical manual of mental disorders-fifth edition (PCL-5) in veterans. Psychol. Assess. http://dx.doi.org/10.1037/pas0000254.

Cao, H., McFarlane, A.C., Klimidis, S., 2003. Prevalence of psychiatric disorder following the 1988 Yun Nan (China) earthquake. Soc. Psych. Psych. Epidemiol. 38 (4), 204-212.

Elhai, J.D., Palmieri, P.A., 2011. The factor structure of posttraumatic stress disorder: a literature update, critique of methodology, and agenda for future research. J. Anxiety Disord. 25 (6), 849-854.

Elhai, J.D., Biehn, T.L., Armour, C., Klopper, J.J., Frueh, B.C., Palmieri, P.A., 2011 Evidence for a unique PTSD construct represented by PTSD's D1-D3 symptoms. J. Anxiety Disord. 25 (3), 340-345.

Elhai, J.D., Miller, M.E., Ford, J.D., Biehn, T.L., Palmieri, P.A., Frueh, B.C., 2012. Posttraumatic stress disorder in DSM-5: estimates of prevalence and symptom structure in a nonclinical sample of college students. J. Anxiety Disord. 26 (1), 58-64.

Fergusson, D.M., McLeod, G.F., Horwood, L.J., 2013. Childhood sexual abuse and adult development outcomes: findings from a 30 year longitudinal study in New Zealand. Child Abus. Negl. 37 (9), 664-674.

Frances, A.J., Nardo, J., 2013. ICD-11 should not repeat the mistakes made by DSM-5. Br. J. Psychiatry 203, 1-2.

Galea, S., Brewin, C.R., Gruber, M., Jones, R.T., King, D.W., King, L.A., et al., 2007. Exposure to hurricane-related stressors and mental illness after Hurricane Katrina. Arch. Gen. Psychiatry 64 (12), 1427-1434.

Hansen, M., Hyland, P., Armour, C., Shevlin, M., Elklit, A., 2015. Less is more? Assessing the validity of the ICD-11 model of PTSD across multiple trauma samples. Eur. J. Psychotraumatol. 6, 28766.

Hyland, P., Shevlin, M., McNally, S., Murphy, J., Hansen, M., Elklit, A., 2016. Exploring differences between the ICD-11 and DSM- 5 models of PTSD: does it matter which model is used? J. Anxiety Disord. 37, 48-53.

Jonas, S., Bebbington, P., McManus, S., Meltzer, H., Jenkins, R., Kuipers, E., et al., 2011.
Sexual abuse and psychiatric disorder in England: results from the 2007 Adult Psychiatric Morbidity Survey. Psychol. Med. 41 (04), 709-719.

Joreskog, K.G., Sorbom, D., 1996. LISREL8 User's Reference Guide. Scientific Software, Chicago.

Kilpatrick, D.G., Resnick, H.S., Milanak, M.E., Miller, M.W., Keyes, K.M., Friedman, M.J., 2013. National estimates of exposure to traumatic events and PTSD prevalence using DSM-IV and DSM-5 criteria. J. Trauma. Stress. 26 (5), 537-547.

King, D.W., Leskin, G.A., King, L.A., Weathers, F.W., 1998. Confirmatory factor analysis of the clinician-administered PTSD Scale: evidence for the dimensionality of posttraumatic stress disorder. Psychol. Assess. 10 (2), 90-96.

Liu, P., Wang, L., Cao, C., Wang, R., Zhang, J., Zhang, B., et al., 2014. The underlying dimensions of DSM-5 posttraumatic stress disorder symptoms in an epidemiological sample of Chinese earthquake survivors. J. Anxiety Disord. 28 (4), 345-351.

Maercker, A., Brewin, C.R., Bryant, R.A., Cloitre, M., Reed, G.M., Ommeren, van, et al. 2013. Proposals for mental disorders specifically associated with stress in the International Classification of Diseases-11. Lancet 381 (9878), 1683-1685.

Murray, J., Ehlers, A., Mayou, R.A., 2002. Dissociation and post-traumatic stress disorder: two prospective studies of road traffic accident survivors. Br. J. Psychiatry 180, 363-368.

Muthen, L.K., Muthen, B.O., 1998-2013. MPlus User's Guide, 7th ed. Muthen \& Muthen, Los Angeles.

Neria, Y., Nandi, A., Galea, S., 2008. Post-traumatic stress disorder following disasters: a systematic review. Psychol. Med. 38 (4), 467-480.

O’Donnell, M.L., Alkemade, N., Nickerson, A., Creamer, M., McFarlane, A.C., Silove, D., et al., 2014. Impact of the diagnostic changes to post-traumatic stress disorder for DSM-5 and the proposed changes to ICD-11. Br. J. Psychiatry 205 (3), 230-235.

Raftery, A.E., 1996. Approximate Bayes factors and accounting for model uncertainty in generalized linear models. Biometrika 83, 251-266.

Schwarz, G., 1978. Estimating the dimension of a model. Ann. Stat. 6, 461-464.

Shevlin, M., Hyland, P., Karatzias, T., Bisson, J.I., Roberts, N.P., 2017. Examining the disconnect between psychometric models and clinical reality of posttraumatic stress disorder. J. Anxiety Disord. 47, 54-59.

Simms, L.J., Watson, D., Doebbeling, B.N., 2002. Confirmatory factor analyses of posttraumatic stress symptoms in deployed and non-deployed veterans of the Gulf War. J. Abnorm. Psychol. 111, 637-647. http://dx.doi.org/10.1037/0021-843X.111.4.637.

Steiger, J.H., 1990. Structural model evaluation and modification: an interval estimation approach. Multivar. Behav. Res. 25, 173-180.

Stein, D.J., McLaughlin, K.A., Koenen, K.C., Atwoli, L., Friedman, M.J., Hill, E.D., et al,, 2014. DSM-5 and ICD-11 definitions of posttraumatic stress disorder: investigating "narrow" and "broad" approaches. Depress Anxiety 31 (6), 494-505.

Tanaka, J.S., 1987. "How big is big enough?": sample size and goodness of fit in structural equation models with latent variables. Child Dev. 134-146.

Tsai, J., Harpaz-Rotem, I., Armour, C., Southwick, S.M., Krystal, J.H., Pietrzak, R.H., 2015. Dimensional structure of DSM-5 posttraumatic stress disorder symptoms: results from the National Health and Resilience in Veterans Study. J. Clin. Psychiatry 76 (5), 546-553.

Tucker, L.R., Lewis, C., 1973. A reliability coefficient for maximum likelihood factor analysis. Psychometrika 38 (1), 1-10.

Watson, D., 2005. Rethinking the mood and anxiety disorders: a quantitative hierarchical model for DSM-V. J. Abnorm. Psychol. 114 (4), 522-536.

Watson, D., 2009. Differentiating the mood and anxiety disorders: a quadripartite model. Annu. Rev. Clin. Psychol. 5, 221-247.

Weathers, F.W., 2017. Redefining posttraumatic stress disorder for DSM-5. Curr. Opin. Psychol. 14, 122-126.

Weathers, F.W., Blake, D.D., Schnurr, P.P., Kaloupek, D.G., Marx, B.P., Keane, T.M., 2013a. The Life Events Checklist for DSM-5 (LEC-5). Retrieved from the National Center for PTSD website: 〈http://www.ptsd.va.gov $\rangle$.

Weathers, F.W., Blake, D.D., Schnurr, P.P., Kaloupek, D.G., Marx, B.P., Keane, T.M., 2013b. The PTSD Checklist for DSM-5 (PCL-5). Retrieved from the National Center for PTSD website: 〈http://www.ptsd.va.gov〉.

Yuan, K.H., Bentler, P.M., 2000. Three likelihood-based methods for mean and covariance structure analysis with non-normal missing data. Sociol. Methodol. 30 (1), 165-200.

Yufik, T., Simms, L.J., 2010. A meta-analytic investigation of the structure of posttraumatic stress disorder symptoms. J. Abnorm. Psychol. 119 (4), 764-776. 